\title{
Multi-level changes to the Japanese electricity grid due to increasing entry of small-scale renewables and alternative fuel vehicles
}

\author{
Kristina Knuepfer ${ }^{1}$, Miguel Esteban ${ }^{1}$, Tomoya Shibayama ${ }^{1}$ \\ ${ }^{1}$ Waseda University \\ 4-1 Okubo 3, Shinjuku-ku, 169-8555 Tokyo, Japan \\ k.knuepfer@asagi.waseda.jp; miguel.esteban@aoni.waseda.jp
}

\section{Extended Abstract}

Electricity grid decarbonisation requires major changes in resource composition, the size and location of power plants, as well as electricity flow direction capabilities [1]. Coupled with transport sector integration, the introduction of different alternative fuel vehicles (AFVs) creates a new demand source that will require distribution, while also offering flexibility balancing capacities to the grid. Japanese grid operators have so far managed regions that are relatively poorly interconnected, resulting in an overcapacity of thermal power stations [2], which negatively affects renewable integration. Regionally, renewable implementation capacities and strategies differ based on a complex set of factors, such as geography, favourable resource type, connectivity with other regions, population developments, car ownership and use, as well as political strategies [3].

The overall aim of this study is to assess the overall maximum renewable integration and associated vehicle technology composition that Japan could support by the year 2030. To do so, three scenarios were formulated, which were compared to a baseline case study of the present-day electricity sector. In doing so, the authors compared the roles and requirements of different AFV technologies as new demand sources and flexibility management tools between metropolitan and non-metropolitan areas, based on the different car ownership and use profiles, as well as resource compositions.

The simulations were carried out using the EnSimGrid model, developed by the authors. This can simulate the hourly supply and demand of electricity of the nine grid operator regions in Japan (excluding Okinawa) together with transmission level interconnection, as well as a parallel hydrogen network. Additionally, in several of these regions the 15 metropolitan areas of the country were separately modelled from non-metropolitan areas. The AFV technologies modelled are fuel cell electric vehicles (FCEVs) and battery electric vehicles (BEVs), with the latter performing vehicle-to-grid services. In all scenarios the renewable generation is based on hourly real-time meteorological data from the JMA, allowing for a realistic simulation of the energy production by these intermittent resources.

The results indicate that there are regional differences between the electricity demand of metropolitan and nonmetropolitan areas that is required for charging AFVs, and therefore different opportunities for renewable integration at different scales (between regions and within different types of areas in the same region). Secondly, by reducing thermal capacities in historical supplier regions to Kanto (in accordance with their regional strategies), such as Tohoku, transmission chains became more spatially distanced from the receiving region. This future expected reduced thermal plant overcapacity provides a compelling incentive to invest in an efficient transmission grid expansion, which renewables would also profit from. Thirdly, a smoothing impact of compatible AFV technology fleets on renewable generation can be observed, especially for BEV fleets in Kyushu (where solar PV is currently dominant), which confirms previous observations regarding vehicle technology and specific resource relationships [4].

\section{References}

[1] M. McPherson and B. Karney, "A scenario based approach to designing electricity grids with high variable renewable energy penetrations in Ontario, Canada: Development and application of the SILVER model," Energy, vol 138, pp. $185-196,2017$.

[2] T. Wakiyama and A. Kuriyama, "Assessment of renewable energy expansion potential and its implications on reforming Japan's electricity system," Energy Policy, vol 115, pp. 302 - 316, 2018. 
[3] G. Trencher and J. van der Heijden, "Contradictory but also complementary: National and local imaginaries in Japan and Fukushima around transitions to hydrogen and renewables," Energy Res. \& Soc. Sci., vol 49, pp. 209 - 218, 2019.

[4] M. Odenberger and M. Taljegard, "Integrating electric vehicles in electricity system models - representing individual driving patterns," $31^{\text {st }}$ Int. Electric Vehicle Symp. \& Exhib. and Int. Electric Vehicle Tech. Conf. Proceedings, Kobe, Japan, 2018. 